\title{
PHOTOSENSITIVE GUEST-HOST POLYMER FOR OPTICAL DATA STORAGE
}

\author{
A. Miniewicz and S. BartKiewicz \\ Institute of Physical and Theoretical Chemistry, Technical University of Wrocław \\ Wybrzeze Wyspiańskiego 27, 50-370 Wrocław, Poland
}

(Received February 23, 1995; in final form April 6, 1995)

Dedicated to Professors Krrysztof Pigoń, Józef W. Rohleder and Zdzistaw Ruziewicz on the occasion of their 70th birthday*

Methylene blue sensitized poly(methyl methacrylate) prepared by the process of dye diffusion has been shown to be efficient medium for recording interference gratings. Phase and/or amplitude holograms can be written in the methylene blue sensitized films of poly(methyl methacrylate) using a conventional source of light a He-Ne laser operating at $632.8 \mathrm{~nm}$ wa velength. Kinetics of photobleaching process of methylene blue is measured. Diffraction efficiencies of up to $30 \%$ were found for thick holograms. Phase conjugation and multiple holograms writing has been tested in the studied system.

PACS numbers: 42.70.Ln, 42.70.Jk, 42.65.Hw

\section{Introduction}

Optical information storage and processing can be realised in materials which exhibit sufficiently strong light-matter interactions. For massive, parallel optical image processing there is a great need for devices in which a spatial light-intensity profile (image) can be recorded and transformed into a transmission or a phase retardation profile. These functions can be performed by non-linear optical materials or photochromic molecules. Among organic materials the best candidates fulfilling the above requirements are polymers with photoactive molecules or molecular assemblies. Various physical and chemical mechanisms can be employed for optical processing among them the important role is played by the molecular photochromism [1]. Widely used holographic techniques of image storage and/or processing rely on photoinduced local changes of material index of refraction. New materials must exhibit reversibility for multiple recording/erasing and fast response

* Submitted on invitation of the Institute of Physical and Theoretical Chemistry, Technical University of Wrocław, Wrocław, Poland. 
for real-time holography or exhibit self-developing properties for conventional holographic purposes.

Methylene blue (hereafter abbreviated as MB), an organic dye has found application in conventional and polarising holography [2]. The principal mechanism of optical recording is photobleaching of MB. Methylene blue sensitized gelatins (MBDCG) [2-10] in which the high diffraction efficiencies of about $80 \%$ were measured are well recognised [6]. The most noticeable drawback is that the formation of these holograms is complex and time consuming procedure including: fabrication of the MBDCG plates, exposure and development [11, 12]. Some trials to put methylene blue into polymer matrix were already reported for acrylamide based photopolymers [13, 14] and polyvinyl alcohol (PVA) [15]. This was possible due to mutual solubility of the polymer and the dye in the same solvent which is the necessary condition to obtain grainless photosensitive holographic material. More recently MB ability for real-time image processing has been experimentally proved by an observation of optical phase conjugation signals in the methylene blue sensitized gelatin films [16] and methylene blue-doped PVA films [15].

In this paper we will describe a new procedure of obtaining MB sensitized poly(methyl methacrylate) (PMMA) matrix (MBPMMA) suitable for holographic recording. MB dye is introduced into pure PMMA matrix by a process of diffusion. We characterize the properties of these materials by showing the results of permanent and transient hologram formations with the use of low power laser source.

\section{Experimental}

\subsection{Sample preparation}

MB (Basic Blue 9, $\mathrm{C}_{16} \mathrm{H}_{18} \mathrm{~N}_{3} \mathrm{SCl}$, Polish Chemicals) without further purification was used for preparation of photosensitive plates. It is well soluble in water and moderately in few organic solvents. For obtaining good optical quality films by casting or spin coating it is necessary to have a solvent which equally well dissolves dye and polymer. The first trials to use solvents such as $\mathrm{CCl}_{4}$, chloroform and acetone have lead to optically nonuniform films containing microcrystallites (up to $30 \mu \mathrm{m}$ in size) which were formed during drying procedure. Our present approach is based on preparation of thin films of PMMA doped with MB on PMMA substrate. The best method for this is to perform dye diffusion into PMMA sample [17]. For investigations we used commercially available $6 \mathrm{~mm}$ thick optical quality finished PMMA plates. We chose methanol as a dye solvent. Methanol is an inert solvent with respect to the PMMA, it can however penetrate into PMMA plate with rate depending on temperature. The processes of dye diffusion were carried out at room temperature and at elevated temperatures up to boiling temperature of methanol. One-side films were obtained by surface protection of another side of the plate during the diffusion process. The final concentration of $\mathrm{MB}$ diffused into PMMA was controlled by changing the time and temperature of the whole process. The optical density of the obtained layers was measured with spectrophotometer (UV-VIS Scanning Spectrophotometer, UV-2101PC, Shimadzu). Samples of transmittance ranging from 0.01 to 0.9 measured at $632.8 \mathrm{~nm}$ were readily obtainable. The desired, for various experiments, optimum transmittances were achieved by 
changing either time or temperature of diflusion process (2 months, $T=298 \mathrm{~K}$, deep dye penetration or several seconds, thin films formation at boiling temperature of methanol). Typical film thicknesses observed by colour change with optical microscope on MBPMMA plates cut perpendicular to film depth ranged from 1 to $50 \mu \mathrm{m}$. These observations allowed us to notice that longer diffusion time yielded films with diffused MB concentration profiles whereas shorter times yielded respectively sharper edges of the dye concentration inside PMMA plate. The exact modified film thickness was however difficult to measure.

\subsection{Experimental techniques}

In order to characterise the effect of light exposure on sample optical properties one needs to know the kinetics of light-induced changes of index of refraction $n$ and absorption coefficient $\alpha$ at least at wavelength of laser light. Both parameters are coupled with each other, then should be measured simultaneously at a given sample spot and light intensity. This can conveniently be done with the help of Mach-Zehnder interferometer. This interferometer was working with a IIe-Ne Spectra Physics 127 laser $(25 \mathrm{~mW} \mathrm{cw}, 632.8 \mathrm{~nm}$ ). Computer controlled light shutter placed at one of the beams allowed to measure both index of refraction changes (shutter in off-state) and absorbance changes (shutter in on-state). Index of refraction changes in function of exposure time can be calculated from recording of moving fringe pattern and absorption changes can be determined from transmittance evolution.

The material holographic performances can be evaluated from measurements of diffraction efficiencies in a self-diffraction experiment i.e. obtained on just written gratings. Two-beam coupling experiment is widely used for interferometric grating formation $[18,19]$. For our studies we employed a standard degenerate two-wave mixing (DTWM) setup using a polarised He-Ne (Carl Zeiss model IINA $188,10 \mathrm{~mW}, \lambda=632.8 \mathrm{~nm}$ ) laser beam. Two equal intensity laser beams

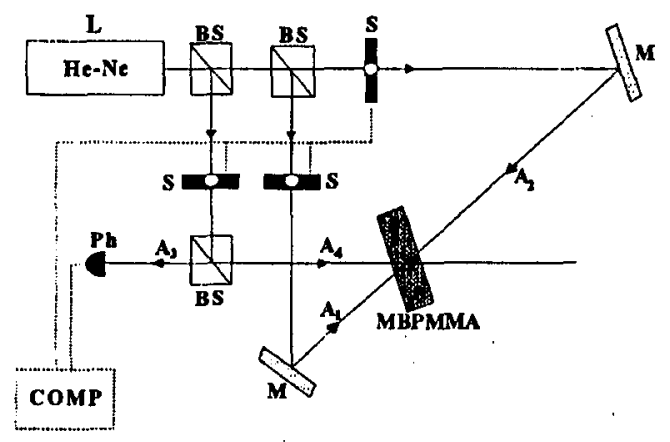

Fig. 1. Experimental setup for studying degenerate four-wave mixing process in MBPMMA layers. $A_{3}$ is a phase conjugate to the probe signal $A_{4} . A_{1}$ and $A_{2}$ are the collinear, antiparallel pumping beams. $L-$ laser, $B S$ - beam splitter, $M$ - mirror, $S$ - light shutter, $P h$ - photodetector, $C O M P$ - computer. 
$\left(I=150 \mathrm{~mW} / \mathrm{cm}^{2}\right)$ were intersected at the sample and at interbeam angles ranging from $3-30^{\circ}$, which allowed for modification of a fringe spacing $\Lambda$. Plates were illuminated in that way that the normal to the surface was parallel to bisectrix of the interbeam angle. Bragg diffraction spots were observed in far field on the screen and measured with a system of calibrated photoresistors. Light intensities at various diffraction orders were monitored and memorised using a multichannel A/D card coupled with a PC computer.

The ability of material to be useful in real-time holography can be checked by observation of phase conjugation phenomenon $[18,19]$. We measured phase conjugation with a modified degenerate four-wave mixing (DFWM) configuration based on linearly polarised He-Ne laser which is shown schematically in Fig. 1. The two counter propagating pump beams $A_{1}, A_{2}$ and the probe beam $A_{4}$ all of similar intensities and apertures of $2 \mathrm{~mm}$, were intersected at the sample. Emerging beam $A_{3}$ antiparallel to $A_{4}$ being its phase conjugate has been measured. Its presence is a proof of a dynamic recording of interference grating within a photosensitive medium.

\section{Results and discussion}

\subsection{Light induced changes of polymer absorption and index of refraction}

In Fig. 2 we present the light induced changes of transmittance of MBPMMA. In this figure the transmitted light intensity is plotted versus time for the same plate but at various incident light intensities equal to $I=300,150$,

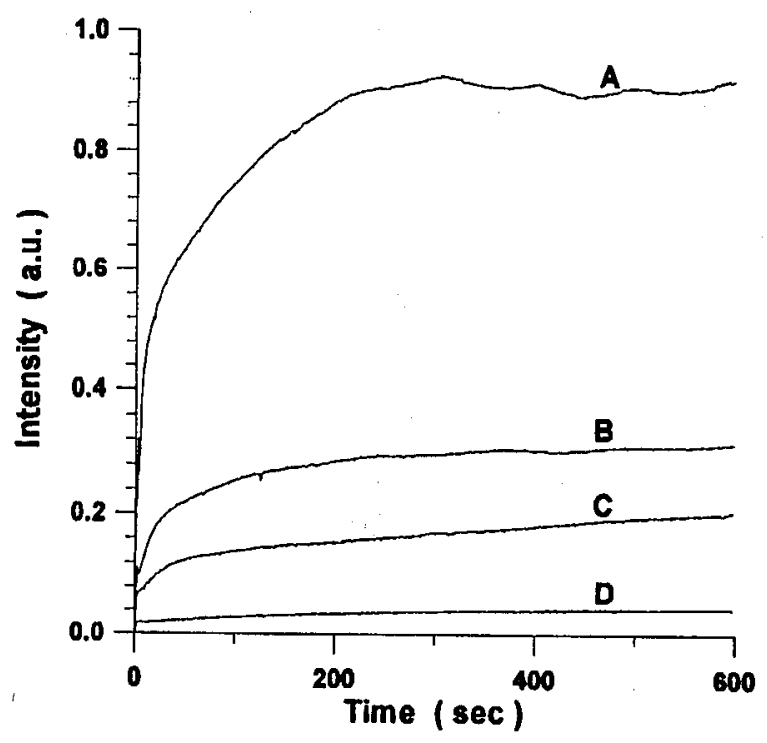

Fig. 2. Bleaching kinetics monitored by output beam intensity vs. time for various input intensities on a MBPMMA plate. Input beam intensities amount to: $A-300$, $B-150, C-75$ and $D-37 \mathrm{~mW} / \mathrm{cm}^{2}$, respectively. 


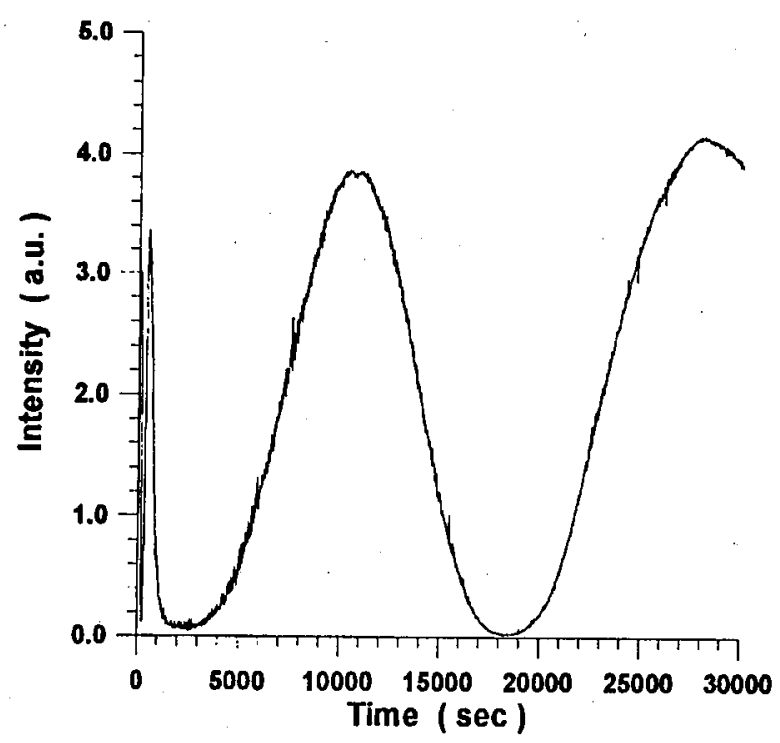

Fig. 3. Measurements of moving interference pattern due to light induced index of refraction changes in MBPMMA layer obtained with Mach-Zehnder interferometer. Phase difference between two consecutive fringe maxima amounts to $2 \pi$.

75 and $37 \mathrm{~mW} / \mathrm{cm}^{2}$, respectively. It is evident that in the MBPMMA samples one deals with a photobleaching process. Light induced reaction transforms the MB molecule into its leuco form (leuco-MB) which does not absorb the light at the excitation wavelength $(632.8 \mathrm{~nm})[2,5]$. The photobleaching rate is strongly dependent on light intensity with tendency of saturation for longer exposure times. For smaller light intensities the saturation seen in Fig. 2 is only an apparent one because the transmitted light intensity increases after prolonged irradiation time almost linearly with a very small slope. The simple model for the real time bleaching process of $\mathrm{MB}$ was developed by Capolla and Lessard [8] for MB sensitized gelatin films. Judging from similar behaviour of transmittance curves presented in [8] and this work one can assume similar bleaching mechanisms most probably connected with demethylation of MB dye.

In Fig. 3 the typically observed shift in time of interference fringes obtained on MBPMMA sample with the help of a Mach-Zehnder interferometer $\left(I=300 \mathrm{~mW} / \mathrm{cm}^{2}\right)$ is shown. From the oscillations of light intensity in function of time one can directly calculate the changes of index of refraction $\Delta n$ of the studied sample providing the thickness $d$ of the sample is known. Between two consecutive maxima there is a phase shift equal to $2 \pi$. Then from the analysis of the curve of Fig. 3 the product $\Delta n \cdot d$ ( $d$ is the thickness of a photosensitive layer) can be calculated in function of time. This is presented in Fig. 4. One can distinguish two regions of index of refraction changes with almost linear time dependencies: initial $(0-350 \mathrm{~s})$ with high slope equal to $2.21 \times 10^{-3} \mu \mathrm{m} / \mathrm{s}$ and subsequent (3000-30000 s) with a small slope equal to $3.78 \times 10^{-5} \mu \mathrm{m} / \mathrm{s}$. From 


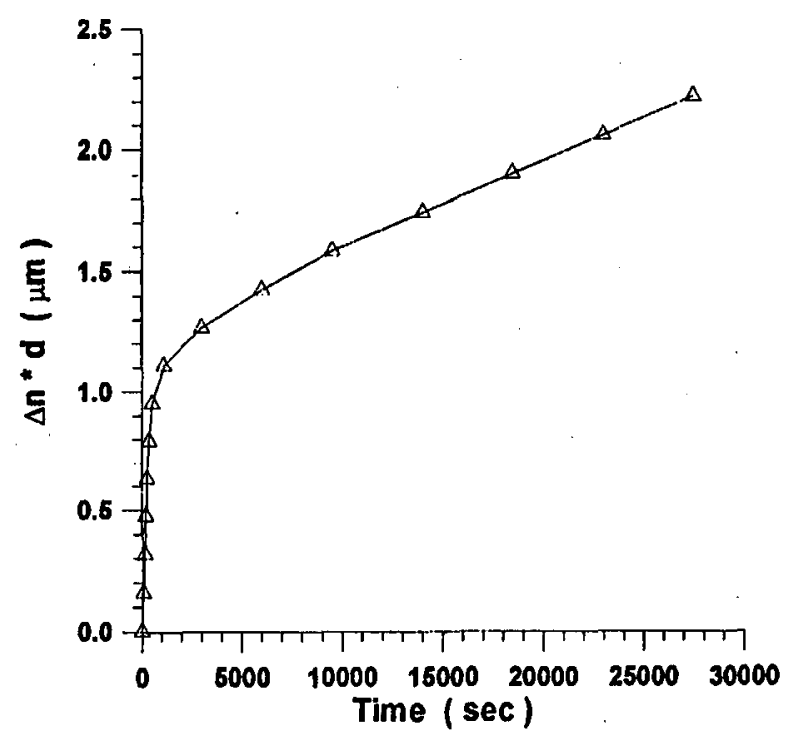

Fig. 4. Calculated from the interference fringe movement optical path $(\Delta n \cdot d)$ changes vs. time for a MBPMMA plate.

the point of view of efficient hologram writing the exposition to light irradiation for the given intensity should last until the beginning of the second region. The absolute values of light induced index of refraction changes $\Delta n$ cannot be determined until the thickness of the photosensitive layer $d$ is not known. The index of refraction for pure PMMA samples (prior sensitization with MB) was determined by the method of total internal reflection and amounts for TE polarization: $1.4913 \pm 0.001$ at $633 \mathrm{~nm}$ and $1.4854 \pm 0.001$ at $810 \mathrm{~nm}$. Diffusion of the dye has not formed a surface wave guiding structure which should be expected for a higher value of index of refraction of the surface layer than that of the bulk of a material. For light wavelength $810 \mathrm{~nm}$ index of refraction of dye sensitized layer amounts to $1.485 \pm 0.001$ which is almost equal to the value measured for pure PMMA.

\subsection{Principle of holographic recording in MBPMMA by two-wave mixing process}

The two-beam coupling technique allows for writing the sinusoidal light interference pattern (grating) formed by two intersecting He-Ne laser beams into MBPMMA sample due to the photochemical reaction of methylene blue which leads to its non-absorbing leuco form. This process known as photobleaching will lead to a spatially modulated photoproduct concentration, thus forming a grating of the complex refractive index $\underline{n}=n+\mathrm{i} \alpha / 2 k$, where $n$ is the index of refraction, $\alpha$ - the absorption coefficient and $k=2 \pi / \lambda$ is the absolute value of the wave vector of the light for which the optical properties are measured. A spatial modulation of the complex refractive index $\underline{n}=n+\Delta \underline{n} \cos (q x)$ where $q$ is the grating wave vector $q=2 \pi / \Lambda$ leads to an amplitude transmittance [18]

$$
t(x)=\exp [\mathrm{i} \phi \cos (2 \pi x / \Lambda)]
$$


where $\phi=2 \pi \Delta \underline{n} d / \lambda$. Generally, two extremal cases can be distinguished: thick and thin gratings which are defined by the value of a parameter $Q$ :

$$
Q=2 \pi d \lambda / \Lambda^{2} n \text {. }
$$

For $Q \gg 1$ one deals with a thick grating whereas for $Q \ll 1$ with a thin one. given by

For thin grating case the amplitudes $I_{m}$ of the $m$-order diffracted waves are

$$
I_{m}=I_{\text {inci }} \mathrm{i}^{m} J_{m}(\phi),
$$

where $J_{m}$ is the $m$-th order Bessel function and $I_{\text {inc }}$ is the incoming light intensity. The diffraction efficiency $\eta=I_{1} / I_{\text {inc }}$ defined as the ratio of 1-order diffracted beam intensity to incident light intensity $I_{\text {inc }}$ is given by [18]:

$$
\eta=\left|J_{1}(\phi)\right|^{2} \text {. }
$$

According to Kogelnik's [20] coupled wave theory the diffraction efficiency $\eta$ for the first order diffracted wave in the case of transmission and thick hologram is given by

$$
\eta=\frac{I_{1}}{I_{\text {inc }}}=\left[\sin ^{2}\left(\frac{\pi \Delta n d}{\lambda \cos \theta}\right)+\sinh ^{2}\left(\frac{\Delta \alpha d}{4 \cos \theta}\right)\right] \exp \left(-\frac{\alpha_{0} d}{\cos \theta}\right)
$$

where $\theta$ is the Bragg angle inside the MBPMMA layer and $\alpha_{0}$ is the average absorption coefficient of the sample. In Fig. 5 we present temporal evolution of 1-order diffracted beam intensity measured during recording of a simple interference grating. The presence of oscillations of diffraction efficiency $\eta$ is theoretically

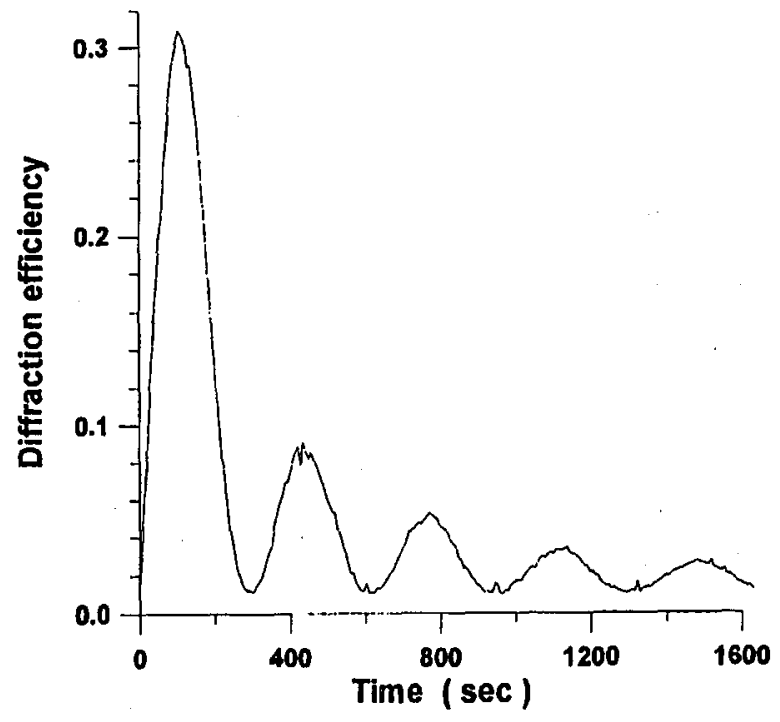

Fig. 5. Temporal evolution of efficiency for 1-order diffracted light recorded during grating formation process in thin layer of MBPMMA. Grating spacing $\Lambda=$ $\lambda / 2 n \sin \left(\theta_{\text {ext }} / 2\right)=5 \mu \mathrm{m}$. 
predicted by Eqs. (4) and (5). For continuous increase in the product $(\Delta n \cdot d)$ with time the phase $\phi(t)$ (cf. Eq. (1)) will also continuously increase with time. The results presented in Fig. 5 clearly indicate that we deal with a diffraction on thin grating within the Raman-Nath regime (cf. Eq. (4)) as the subsequent maxima of $I_{1}$ diminish with time. Closer inspection of the obtained results leads us to conclusion that the phase grating contribution is dominant (cf. Ref. [18], p. 99, Fig. 4.3) which means that the ratio $\Delta \alpha \lambda / \Delta n 4 \pi \leq 0.1$ for the studied sample. The maximum diffraction efficiency in the presented experiment reached after $150 \mathrm{~s}$ a value equal to $c a .30 \%$ which is close to the theoretically predicted limit.

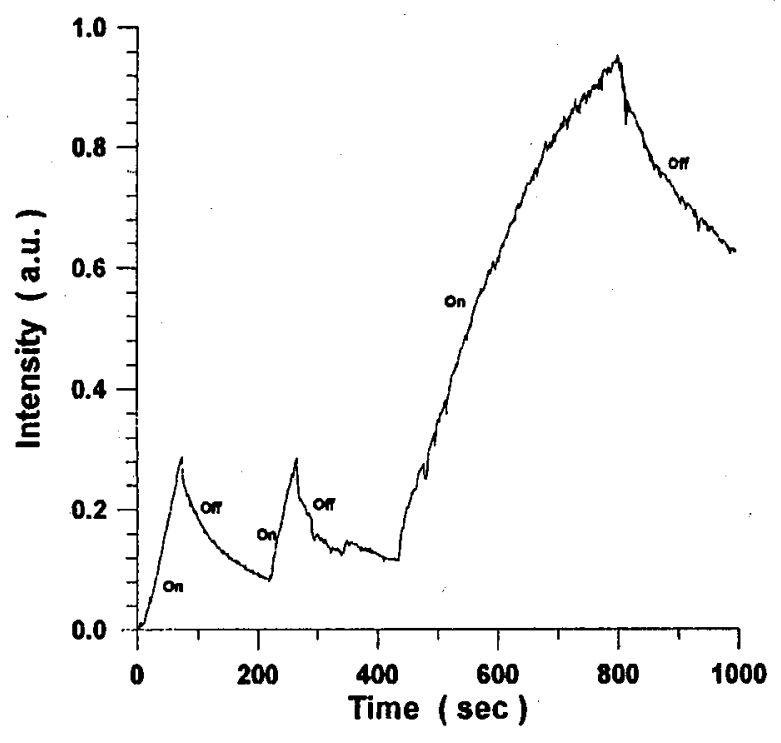

Fig. 6. 1-order diffracted light intensity measured during sequential hologram writing (two beams on) and optical erasing (one beam off) in MBPMMA sample.

In Fig. 6 we show an example of writing several subsequent holograms in the same spot of a sample. By reducing the recording time and/or incident light intensity one does not reach the large $\phi$ values thus there is a possibility to record more than one hologram at the same sample spot. The recording rate seen in Fig. 6 exceeds erasing one mainly due to the fact that the erasing is performed simply by cut-off of one of the laser beams (the half of the power, off-state in Fig. 6). Under the same experimental conditions it was observed that the initial hologram recording speed is higher at elevated temperatures. We have also successfully check the possibility of recording of multiple sinusoidal interference gratings in the same sample spot using different Bragg angles during writing procedure.

\subsection{Phase conjugation in MBPMMA}

The dynamic formation of gratings is crucial for real-time holography and can be realised in media with sufficiently fast material response to illumination pattern. 


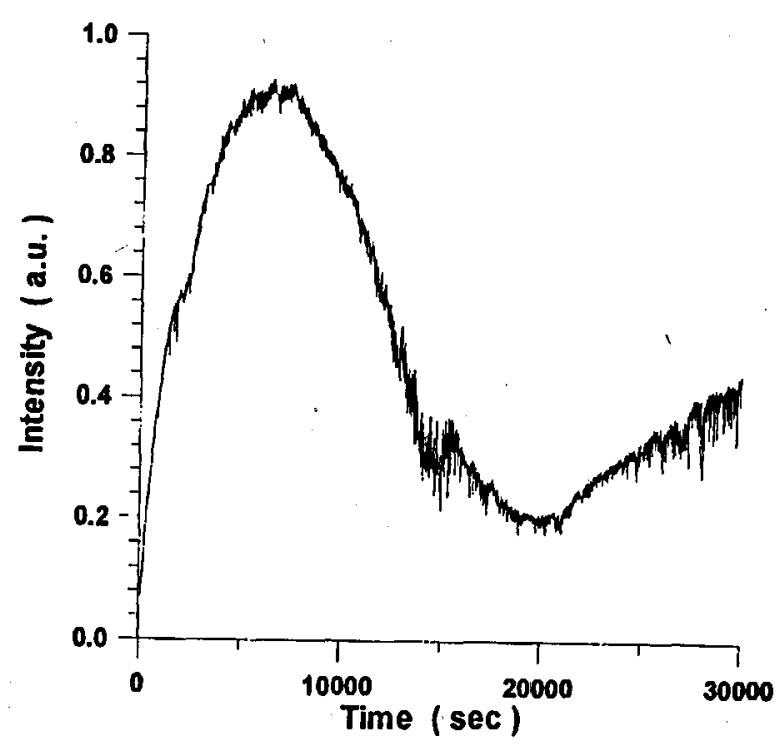

Fig. 7. An example of phase conjugate signal obtained in DFWM experiment in MBPMMA vs. time.

The experimental manifestation of the dynamic grating formation is the process of generation of a phase conjugate beam which arises as a result of a degenerate four-wave mixing process [21]. Phase conjugation phenomenon in MB sensitized gelatin was already described by us [22], here we would like to show (cf. Fig. 7) that the process can also be observed in MBPMMA samples. Phase conjugate reflectivity obtained for this system amounted to $1 \%$. One should however mention that the main contribution to the total phase conjugate response comes from the holographic (permanent) gratings and the contribution of transient (real-time) gratings such as excited state population grating is negligible.

\section{Conclusions}

We have shown that methylene blue sensitized poly(methyl methacrylate) system can be applied for recording an interference pattern using a weak power $\mathrm{He}-\mathrm{Ne}$ laser as the light source. Phase diffraction gratings were obtained in thin layers of MBPMMA without application of any development procedure. 1-order diffraction efficiency amounting to $30 \%$ reached almost a theoretical limit predicted for light diffraction on thin phase holograms. The presented technique allows for sensitization of commercial PMMA plates with other organic dyes (e.g. phenothiazine derivatives, triphenylmethane dyes) or their mixtures (e.g. Giemsa and May-Grünwald stains). Low cost of preparation of photosensitive holographic recording films, very simple holographic recording process and possibility of tailoring of properties of photosensitive layers make possible many commercial applications for the PMMA based holographic recording materials. 


\section{Acknowledgments}

We would like to thank Dr. M. Samoć for measurements of index of refraction in PMMA. This work was sponsored by the Technical University of Wroclaw.

\section{References}

[1] B.L. Feringa, W.F. Jager, B. de Lange, Tetrahedron 49, 8267 (1993).

[2] T.A. Shankoff, Appl. Opt. 7, 2101 (1968).

[3] B.J. Chang, C.D. Leonard, Appl. Opt. 18, 2407 (1979).

[4] T. Kubota, T. Ose, M. Sasaki, K. Honda, Appl. Opt. 15, 556 (1976).

[5] C. Solano, R.A. Lessard, P.C. Roberge, Appl. Opt. 26, 1989 (1987).

[6] T. Mizuno, T. Goto, M. Goto, K. Matsui, T. Kubota, Appl. Opt. 29, 4757 (1990).

[7] O. Salminem, T. Keinonen, M. Pellinen, Opt. Commun. 70, 277 (1989).

[8] N. Capolla, R.A. Lessard, Appl. Opt. 30, 1196 (1991).

[9] R. Changkakoti, S.V. Pappu, Appl. Opt. 28, 340 (1989).

[10] N. Capolla, R.A. Lessard, Appl. Opt. 27, 3008 (1988).

[11] T. Mizuno, T. Goto, M. Goto, K. Matsui, T. Kubota, Proc. SPIE 1212, 40 (1990).

[12] S. Calixto, R.A. Lessard, Appl. Opt. 23, 1989 (1984).

[13] D.H. Close, A.D. Jacobson, J.D. Margerum, R.G. Brault, F.J. McClung, Appl. Phys. Lett. 14, 159 (1969).

[14] N. Sadlej, B. Smolinska, Opt. Laser Technol. 7, 175 (1974).

[15] II. Nakatasuka, D. Masouka, T. Yamamoto, S. Uemura, R. Yano, Nonlinear Opt. 1, 125 (1991).

[16] A.T. Regunath, C.K. Subramanian, P.S. Narayanan, M.R. Sajan, Appl. Opt. 31, 4905 (1992).

[17] S. Bartkiewicz, A. Miniewicz, Appl. Opt. (1995), to be published.

[18] H.J. Eichler, P. Günter, D.W. Pohl, in: Laser-Induced Dynamic Gralings, Springer Series in Optical Sciences, Vol. 50, Springer-Verlag, Berlin 1986.

[19] P. Günter, J.-P. Huignard, Eds. in: Pholorefractive Materials and their Applications I, Topics in Applied Physics, Vol. 61, Springer-Verlag, Berlin 1988.

[20] H. Kogelnik, Bell. Syst. Tech. J. 48, 2909 (1969).

[21] H. Jagannath, H.J. Caulfield, in: Modern Nonlinear Optics, Eds. M. Evans, S. Kielich, Vol. 85, Part 2, in series Advances in Chemical Physics, Wiley, New York 1993 , pp. $1-50$.

[22] S. Bartkiewicz, A. Miniewicz, Nonlinear Opt., 1995, to be published. 